\title{
Chapter 7 \\ Visualize and Communicate Extreme Weather Risk to Improve Urban Resilience in Malawi
}

\author{
Alessandro Demarchi, Elena Isotta Cristofori and Anna Facello
}

\begin{abstract}
Since the last century, an unprecedented settlement expansion, mainly generated by an extraordinary world population growth, has made urban communities always more exposed to disasters. Casualties and economic impacts due to hydro-meteorological hazards are dramatically increasing, especially in developing countries. Although the scientific community is currently able to provide innovative technologies to accurately forecast severe weather events, scientific products are often not easily comprehensible for local stakeholders and more generally for decision makers. On the other hand the integration of different layers, such as hazard, exposed assets or vulnerability through GIS, facilitates the risk assessment and the comprehension of risk analysis. This work presents a methodology to enhance urban resilience through the integration into a GIS of satellite-derived precipitation data and geospatial reference datasets. Through timely and meaningful hydro-meteorological risk information, this methodology enables local government personnel and decision makers to quickly respond and monitor natural phenomena that could impact on the local community. This methodology is applied to the January 2015 Malawi Flood case study and result will be discussed along with further recommended developments.
\end{abstract}

Keywords Flood • Urban resilience • Weather risk

\footnotetext{
A. Demarchi $(\bowtie) \cdot$ E.I. Cristofori · A. Facello

TriM-Translate into Meaning, Corso Sommeiller 24, 10128 Turin, Italy

e-mail: alessandro.demarchi@trimweb.it

E.I. Cristofori

e-mail: elena.cristofori@ trimweb.it
}

A. Facello

e-mail: facelloanna@gmail.com 


\subsection{Introduction}

The course of the XX century has been characterised by two unprecedented demographic phenomena. Firstly, an exceptional growth of world population which is currently more than four times compared to the beginning of the last century (UNDESA 2011). Secondly, the rise of the urban population which in 2010 has exceeded the rural population for the first time in the history (UNDESA 2011).

Combined together, these two demographic trends have determined a rapid expansion of either planned or informal urban areas. All over the world, to meet this insatiable demand for land, communities were settled also in dangerous areas prone to natural disasters. Indeed, new settlements and infrastructures have been built on active faults, under landslides or mudslides, in flooding areas, in areas of volcanic eruptions, etc. This unprecedented urban sprawling phenomenon has involved both developed and developing countries. On the other hand, increases in frequency and intensity of extreme weather events, mainly due to variation either in the mean state of the climate or in its variability, are posing an always more serious risk to worldwide urban communities (Teegavarapu 2012; IPCC 2012). Indeed, even if there is still some uncertainty about the rates of yearly change and it is difficult to distinguish between extreme events exacerbated by climate change from those that represent part of the current natural variability of climate, the Intergovernmental Panel on Climate Change (IPCC) identifies climate impacts as one of the most relevant environmental issues over the 21st century (Cardona et al. 2012; IPCC 2014).

As a consequence, the increased likelihood of urban communities to be affected by natural events, along with climate change effects, is always more frequently causing large-scale disasters. Indeed, over the last century, the number of reported calamities due to natural phenomena has dramatically risen (CRED and UNISDR 2016; Tiepolo and Cristofori 2016b).

Among all natural phenomena, the number of disasters related to hydrometeorological events is predominant. According to CRED and UNISDR (2016), the overwhelming majority of natural disasters is indeed due to floods, storms and other weather-related events. These phenomena are clearly affecting the largest number of people and they are causing the largest amount of economic damage among all the different typologies of phenomena. Only over the last twenty years, EM-DAT recorded 6457 weather-related disasters which claimed 606,000 lives and 4.1 billion people injured (CRED and UNISDR 2016).

Considering the high level of exposure and the high level of vulnerability due to unprepared human communities, hydro-meteorological phenomena are particularity dangerous in developing countries where these events are causing always more massive impacts in terms of causalities, economic and environment damages (Tiepolo and Cristofori 2016b). According to United Nations Office for Disaster Risk Reduction (UNISDR), Disaster Risk Reduction (DRR) aims to reduce the damage caused by natural hazards like floods, droughts, cyclones, etc., through an ethic of prevention. DRR framework includes on the one hand, long-term actions, such as a more adequate urban and territorial planning to guarantee a sustainable 
development and to make the local communities safer. On the other hand, DRR includes also short and medium-term actions, such as undertaking prevention and preparedness actions in order to reduce damage due to approaching hazardous phenomena (Eiser et al. 2012).

Nowadays, scientific community is able to provide innovative technologies to accurately forecast hazard weather phenomena. However, results coming from weather forecast must be translated into other languages, to be operationally usable by decision makers. As a matter of fact, within this translation a critical role is mainly played by two actors. The first actor is the scientific community, who produces numerical results, offers their interpretation and gives an assessment of the uncertainty. The second actor is represented by the users who have to understand the scientifically based information and use them to make decisions. Therefore the representation of the final risk information in an understandable, meaningful and usable manner is a key issue in order to implement a proper DRR strategy. This issue is particularly related to the temporal and spatial scale at which the phenomena is studied and to the end-users needs or competencies.

With the aim of implementing effective actions to enhance adaptation and resilience, the increase of awareness about severe hydro-meteorological events through an improved access to technologies is considered essential (UNISDR 2015). In this framework, Geospatial Information Technologies offer a broad assortment of innovative solutions in many different sectors and activities such as: Disaster Risk Management (DRM) and DRR (Altan et al. 2013; Miyazaki et al. 2015; Cristofori et al. 2016a), assessment of environmental issues (Melesse et al. 2007; Chigbu and Onukaogu 2013; Bonetto et al. 2016) and climate change analyses (Donoghue 2002; Sundaresan et al. 2014; Tiepolo and Cristofori 2016a). In particular, Geographic Information Systems (GIS) offer an essential environment for the effective collection, management, representation and multidisciplinary analysis of miscellaneous geographic data (Tomaszewski 2014). The integration and visualization of various data derived from different sources into one system, permits to provide immediately understandable cartographic products that can address a variety of issues such as potential impacts due to hydro-meteorological phenomena on land use, health care facilities, transportation infrastructures or households (Ferrandino 2014; Duncan et al. 2014; Cristofori et al. 2016b). In addition, Remote Sensing (RS), providing timely and reliable information about the Earth's surface and atmosphere, is a primary method for observing weather and climate dynamics (Cristofori et al. 2015), while technical and non-technical users may accurately define the position of relevant environmental and geographic features through Global Positioning System (GPS) tools and applications. Finally, geospatial information technologies allow to effectively reduce costs, both in term of time and economic resources required for carrying out in situ spatial surveys (Cay et al. 2004). These technologies indeed permit to access a big amount of information characterized by a large spatial and temporal coverage and, at the same time, they provide the possibility to easily update the data for monitoring activities (Pandey and Pathak 2014). 
The main objective of this paper is therefore to present a multidisciplinary approach to strengthen urban resilience by creating a GIS in which integrate, manage and visualize hazard information along with general reference data coming from different sources. Through an enhanced accuracy and usability of extreme precipitation warnings, this methodology aims to enable local government personnel and decision makers to quickly respond and monitor natural phenomena that could cause an impact for the local community.

In the following paragraphs will be presented, firstly the data used and the methodology, secondly the description of the case study (the 2015 Malawi flood event), thirdly the obtained results, and finally the conclusions and some future developments.

\subsection{Data and Method}

The proposed methodology is an operative development of a previously published work (Cristofori et al. 2015). One of the principal aims was to properly monitor and forecast extreme rainfall and the potential related physical impacts on populated places. A methodology to easily process satellite-derived precipitation data for the assessment of extreme precipitation hazard was presented along with the application of the methodology to a past event (2013 Malawi Flood). Therefore data used to perform the monitoring and forecasting of extreme precipitation within this paper are the same used in the work presented by Cristofori et al. (2015).

Since the possibility to immediately and easily identify lifelines, infrastructure and facilities affected may effectively supports decision-makers, emergency and recovery teams, additional datasets concerning exposure information have been considered in this work. In this framework, the development of a tailored GIS represents an effective tool to easily and quickly visualize, analyse and integrate into the same system, hazard information with general reference data. The flexibility of the GIS environment allows indeed to easily updating and adding many typologies of data coming from different sources, such as raster satellite-derived precipitation and numerical weather prediction-Hazard data-and geospatial reference vector datasets-Exposure data.

Thus, this methodology allows end-users to adapt the warning messages to the profile and to the specific characteristics of the geographic features (e.g. populated places, infrastructures, facilities, cultivated and grazing areas). In this way, both before and in the aftermath of a hydro-meteorological event, the areas and the resources affected may be identified more precisely and more effective warning messages may be generated. The possibility to issue different and tailored warning messages represents one of the principal innovative development presented in this work.

Moreover, since this methodology aims to be economically sustainable, it has been generated using only open-source data in an open-source environment (QGIS software, released version 2.8.3). Data used for the hazard assessment are indeed 
two open-source rainfall products derived from satellite precipitation estimates and from numerical weather prediction model: the near-real time version of the Tropical Rainfall Measuring Mission (TRMM) 3B42RT and the Global Forecast System (GFS). TRMM (period of record 1997-2015), was a joint satellite mission of NASA in cooperation with the Japan Aerospace Exploration Agency (JAXA) which covered the latitude band between $50^{\circ}$ North and $50^{\circ}$ South; it was designed to study tropical rainfall for weather and climate research. Data are available at $0.25^{\circ} \times 0.25^{\circ}$ spatial resolution, with $3 \mathrm{~h}$ revisit time (Huffman et al. 2007, 2010). TRMM was mainly used to monitor the evolution and magnitude of extreme precipitation events in real-time. Actually, a new joint mission, the Global Precipitation Measurement (GPM), developed by the same agencies and based on the notable successes of the TRMM, is operative.

GFS is a weather forecast model produced by the National Centers for Environmental Prediction (NCEP) of United States. The dataset covers the entire world and it contains several atmospheric and land-soil variables such as temperatures, winds, and precipitation to soil moisture. GFS may be used to operationally forecast an event up to 16 days in advance. Data are available at $0.5^{\circ} \times 0.5^{\circ}$ spatial resolution, with $3 \mathrm{~h}$ revisit time.

TRMM is mainly used to monitor the evolution and magnitude of extreme precipitation events in real-time while GFS supports the prediction of possible hazardous weather phenomena. Therefore a complementary use of these two products represents a fundamental tool for local governmental personnel and decision makers because it allows to predict in advance and to monitor the temporal and spatial evolution of both short-term heavy rainfall and medium-term persistent rainfall events.

Data used for exposure assessment have been acquired from open-source web portals, such as OpenStreetMap (OSM), Geonode, Global Administrative Areas Database (GADM), FAO Geonetwork. Therefore, depending also on the specific end-user needs, several data have been downloaded, processed and integrated into the system. Nonetheless, if the availability of geospatial datasets, needed to assess areas, proprieties or population most at risk are only partial, not-updated or even denied, extraction techniques may be performed to acquire further information from earth observation data (Ehrlich and Tenerelli 2012; Bonetto et al. 2016).

The main phases of the proposed methodology are hazard assessment, integration of exposure information, and generation of risk warnings.

\subsubsection{Hazard Assessment}

The first step to assess the magnitude of hazardous hydro-meteorological events is the calculation of accumulated precipitations over the area of interest. Several time frames may be chosen to provide hazard information concerning the typology of event (i.e. 6-24 h for short-term heavy rainfall and 2-7 days for persistent rainfall). 
This operation is performed in GIS environment using a specific in-house tool which allows to cumulate for each pixel the relative values of the 3-h TRMM precipitation products covering the time span considered.

Once calculated the cumulated precipitation, the second step is the determination of extreme precipitation thresholds, which may be defined as the amount of rainfall that may trigger a "disaster" (such as flooding, landslides, etc.). Consequently, once a given threshold is exceeded, a warning is issued by the alert system. The values of thresholds have to be defined in advance in order to deliver a warning message when a hazardous hydro-meteorological event is approaching and/or affecting a specific geographic area. Different approaches exist to calculate adequate thresholds and the scientific community is still conducting investigations on this topic. In this methodology, a statistical meteorological approach has been followed in order to derive national and regional percentile values. Processing the after-real-time TRMM historical dataset (from 1998 to 2015), the 95th, 97th and 99th percentile have been calculated for different accumulated precipitation timeframes and have been chosen as low, medium and high alert levels.

\subsubsection{Integration of Exposure Information}

Hazard information derived from satellite precipitation estimates or from numerical weather prediction models are often represented using images that give only a rough idea of the potential impact of a flood. Being expressed using raster or gridded format, the rainfall values are indeed returned on pixel scale and the understanding of such information can be particularly difficult for generic and non-scientific users (Cristofori et al. 2015). Moreover, in order to shift from "pure" hazard information to a more "comprehensive" risk conception, the assessment of exposed features has to be as well considered and integrated in the system.

In order to identify the potentially most affected features due to an approaching event in the near-real-time after a disaster, the overlapping between the hazard information assessed and the exposure layers is performed in the created GIS. In particular, the following georeferenced layers are considered:

- administrative subdivision, to better contextualize the affected area;

- census data expressed on administrative area, to assess the number of population potentially affected;

- built-up areas and villages, to identify which human settlements are affected;

- infrastructures (i.e. road network, rail network, train stations, airports and harbours), to identify the best routes for emergency and recovery teams avoiding possible network interruption;

- facilities (i.e. school, religious, government and health-care buildings), to address emergency and recovery teams, means, resources and eventually rescued people avoiding facilities at-risk and/or already affected; 
- land-use/land-cover characterization, to know the typology of affected areas;

- natural resources (i.e. lakes and rivers), to identify further hazardous sources which may contribute to generate cascading effects related to hydrometeorological events.

\subsubsection{Generation of Risk Warnings}

The third and last phase of the proposed methodology regards the generation of risk warning messages. As previously described, once a given threshold is exceeded, a warning is issued by the alert system. Depending on the magnitude of hydro-meteorological phenomenon, the warning message may be differentiated in three levels of alert. A set of thresholds, composed by different crescent values corresponding to each level of resulting warning, has been defined. In this way, when the value of rainfall forecasted or monitored is exceeding the thresholds based on TRMM time series, the corresponding alert level is issued as following:

- Low alert. Precipitation $>95$ th percentile

- Medium alert. Precipitation $>97$ th percentile

- High alert. Precipitation $>99$ th percentile

Applying this 3-level alert system to administrative areas, human settlements, infrastructure and facilities, several typologies of information may be generated in the form of easy to understand final risk maps. These cartographic products allow to meaningful and immediate visualize the areas and the assets affected or at the risk according to the level of risk warning issued.

\subsection{Case Study}

As previously stated, this methodology has been conceived as a low-cost tool sustainable also for developing countries which are very often characterized by a shortage both in term of money and personnel. Therefore, in order to test and validate the methodology developed, the 2015 Malawi flood has been chosen as case study. Since December 2014, exceptional heavy rains affected Malawi causing rivers to overflow and floods. More than $400 \mathrm{~mm}$ and more than $500 \mathrm{~mm}$ were recorded respectively on one and three days of cumulative rainfall which represent the highest measurements since the establishment of meteorological stations in the highlands area of the county (i.e. the Chichiri station in Blantyre) (Fig. 7.1).

Between the 10th and the 12th of January 2015, 15 Malawi districts (out of 28), and in particular the southern districts such as Nsanje, Chikwawa, and Phalombe, 

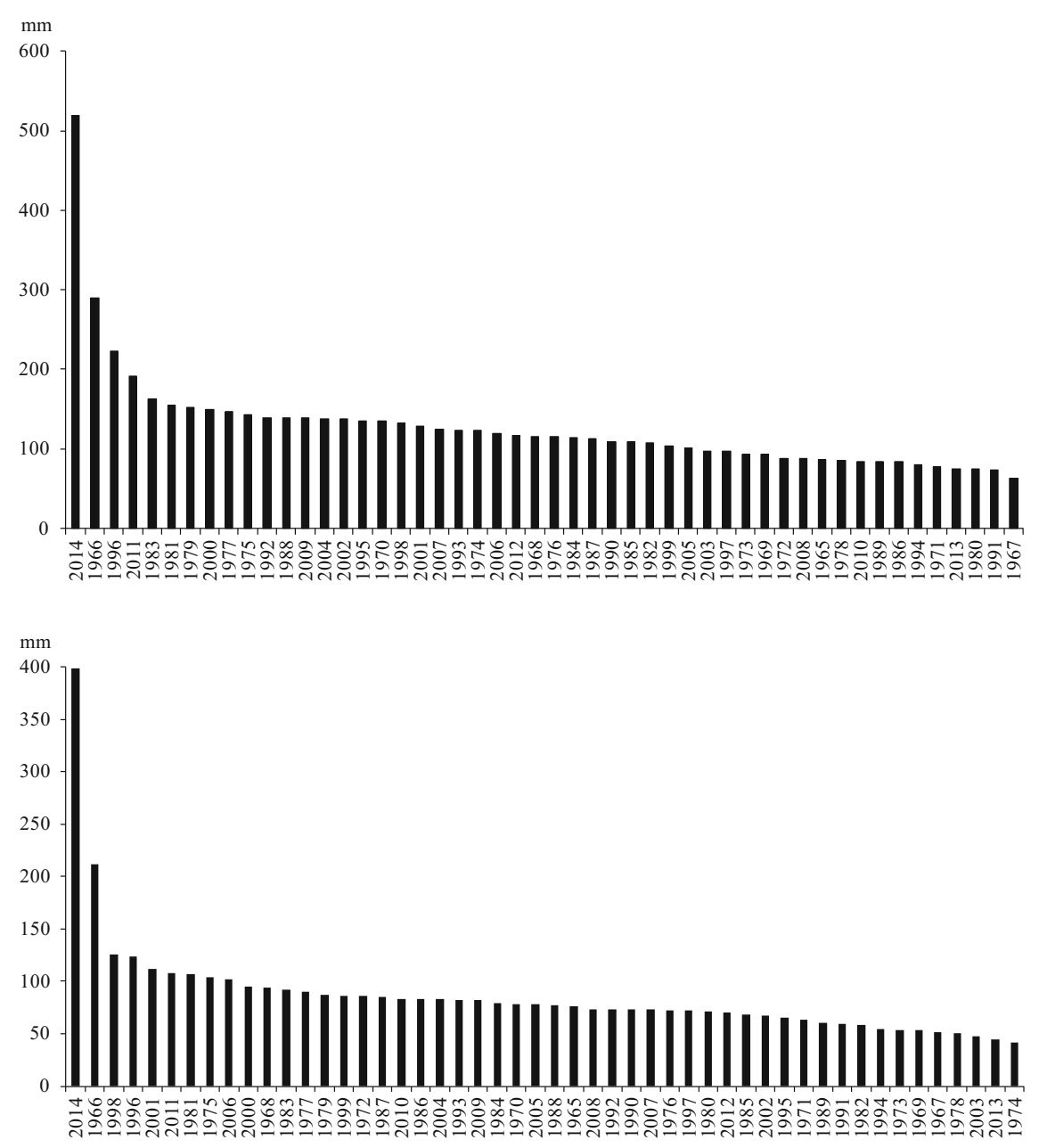

Fig. 7.1 Cumulative rainfall recorded in the Chichiri meteorological station since its establishment until December 2014; max 3 day rainfall (top) and max 1 day rainfall (bottom) in order of magnitude

were hit by severe flooding, affecting more than 1 million people and killing 106 person. Extensive damages to houses, infrastructures (i.e. roads, bridges, water and power supply) and facilities (i.e. schools) were recorded. Considering that several crops of maize were destroyed and livestock killed, the economic system of Malawi, essentially based on subsistence farming, was severely impacted (Government of Malawi 2015). 


\subsection{Results}

In this case study a set of monitoring activities, which could have been performed by government personnel and decision makers both during and in the aftermath of the extreme event, have been simulated. The results of this simulation are presented and discussed in this section.

Following the methodology workflow described in the paragraph 7.2, the first operation performed has regarded the calculation of the accumulated precipitations recorded by TRMM satellite sensor over the selected $72 \mathrm{~h}$ (from 00:00UTC $10 / 01 / 2016$ to $00: 00$ UTC $13 / 01 / 2016$ ) resulting as mainly characterized by heavy rainfalls. The south-eastern part of the country appears to have been particularly affected since several pixels show values greater than $200 \mathrm{~mm}$ of precipitation over the selected period (Fig. 7.2a). Considering that satellite measurements are expressed on a square matrix of $0.25^{\circ} \times 0.25^{\circ}$, the precipitation estimates are provided as mean values equally distributed over the area of each pixel. For this reason, these values may differ from single ground measurements recorded on-site by the meteorological stations.

Comparing accumulates precipitations with thresholds values, gridded alerts have been derived (Fig. 7.2b). It is possible to observe that almost all the southern areas of the country would have been interested by a general state of alert ranging

(a)

(b)

(c)

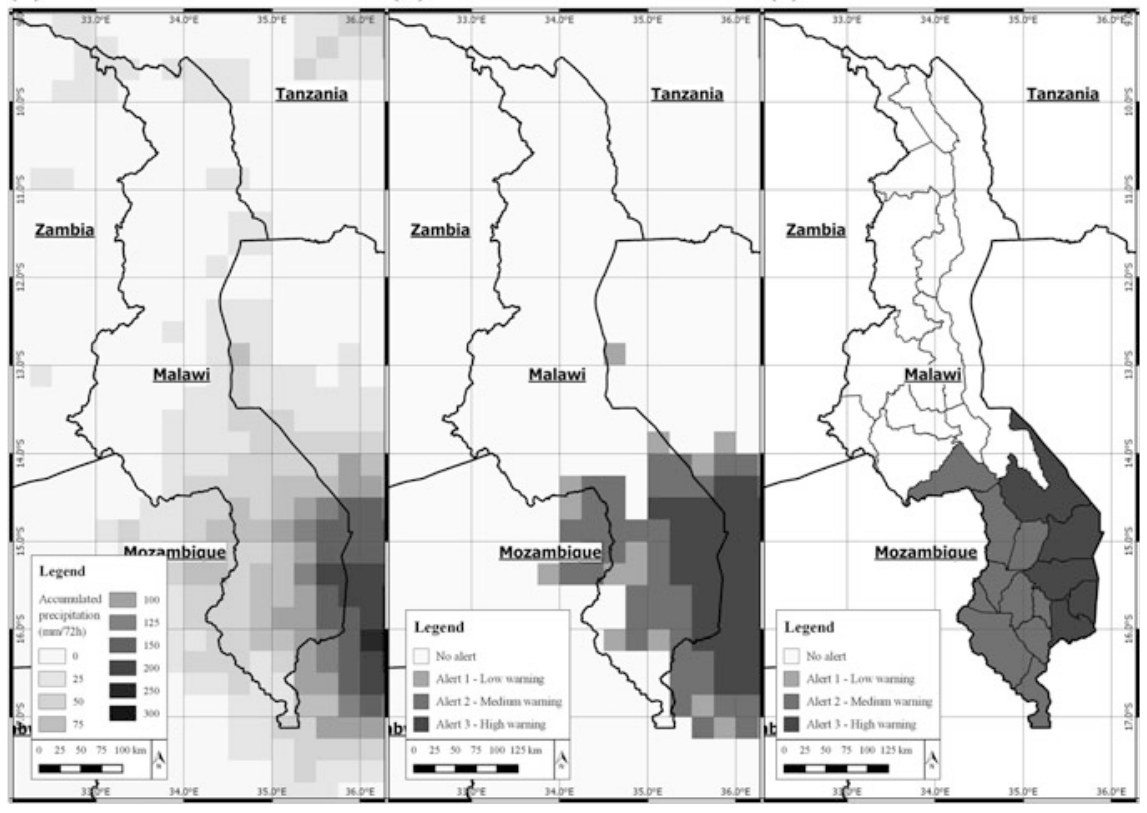

Fig. 7.2 $72 \mathrm{~h}$ accumulated precipitation based on TRMM-3B42RT issued the 12th January 2015 (a), extreme precipitation alerts (b) and administrative districts alerted (c) 
from low alert to high alert messages. On the one hand, this cartographic product could be very useful especially for depicting a first overall description of the areas potentially most affected and for describing the dynamic and the magnitude of the hydro-meteorological event.

On the other hand, an adequate geographic contextualization with an assessment of geographic features, people or infrastructures potentially mostly affected would help to drive decisions during both prevention and response phases of a disaster. Indeed the integration of administrative borders in the system allows to immediately pinpoint the districts mainly affected (Fig. 7.2c). Moreover the GIS application enables an immediate quantification of possible affected population (Table 7.1).

The second step of the methodology is devoted to enhance the understandability and usability of extreme precipitation warnings by highlighting the places and assets potentially most at risk. In the framework of the Shire River Basin Management Program, the Government of Malawi together with the World Bank developed on top of the GeoNode application the Malawi Spatial Data Platform (MASDAP) (Balbo et al. 2013). Therefore several data of potentially affected features such as populated places (i.e. villages and towns), the road network, the health-care facilities (i.e. hospital) and the educational buildings (i.e. schools and universities) have been downloaded from the MASDAP platform. The downloaded layers have been then uploaded into the GIS and integrated with the extreme precipitation alerts.

The intersection of the populated places layer with the extreme precipitation alerts layer allows to immediately identify villages and towns alerted within each district. An alert level has been associated to the villages contained within each pixel of the gridded extreme precipitation alerts layer. In this way, specific alerts

Table 7.1 Population potentially affected per administrative district

\begin{tabular}{l|l|l|l}
\hline District & Alert level & Population & $\begin{array}{l}\text { Population density } \\
\text { Inh/km }\end{array}$ \\
\hline Balaka & 2 & 349,121 & 164 \\
\hline Blantyre & 2 & 364,708 & 204 \\
\hline Chikwawa & 2 & 475,140 & 97 \\
\hline Chiradzulu & 2 & 301,586 & 396 \\
\hline Dedza & 2 & 671,137 & 179 \\
\hline Mwanza & 2 & - & - \\
\hline Neno & 2 & 124,430 & 79 \\
\hline Nsanje & 2 & 255,995 & 132 \\
\hline Ntcheu & 2 & 513,865 & 158 \\
\hline Thyolo & 2 & 603,129 & 361 \\
\hline Machinga & 3 & 538,345 & 137 \\
\hline Mangochi & 3 & 885,355 & 132 \\
\hline Mulanje & 3 & 543,745 & 272 \\
\hline Phalombe & 3 & 338,219 & 238 \\
\hline Zomba & 3 & 614,268 & 197
\end{tabular}


have been addressed to each village in relation to the amount of precipitation measured in the surrounding of their location which roughly corresponds to a square area of $750 \mathrm{~km}^{2}$. A map produced for the southern Malawi highlights the alerts calculated considering the populated places (Fig. 7.3b). The analysis performed so far takes into account only the hazard layer as a criterion to calculate the extreme precipitation warnings. On the other hand the calculation of exposure allows the identification of assets most at risk and a most effective prioritization of emergency actions. Therefore the proximity to the main river network has been considered as an exposure criterion to perform the third step of the proposed methodology. This criterion aims to:

- highlight the risk of those settlements lying within $1 \mathrm{~km}$ from the riverbanks-in this case the alert level has been incremented by one level without trespassing the maximum level (villages characterized by a high alert did not change the level)

- conceal the risk of those populated places located far away from the riverbanks -in this case the alert level has been decremented by two levels

The application of this exposure criterion allows emergency managers and local government personnel to better manage their resources and means. Through the

(a)

(b)

(c)

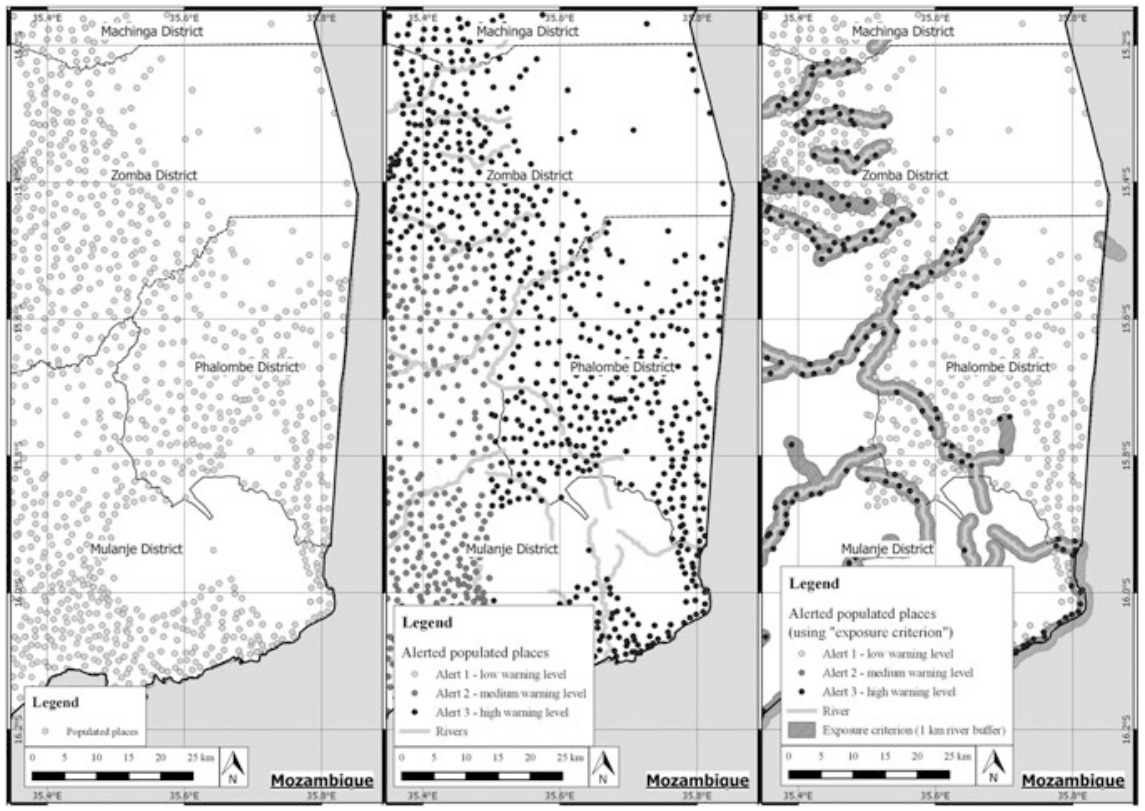

Fig. 7.3 Populated places in Phalombe district, Malawi (a), alerted settlements issued for 12th January 2015 on the basis of extreme precipitation alerts in Phalombe district (b) and alerted settlements issued for 12th January 2015 on the basis of the "exposure criterion" (c) 
identification of most affected areas, they may prioritize their actions directly to those villages characterized by the greater alerts (Fig. 7.3c).

Further analyses have been then performed in order to identify potential impacts on urban and local facilities due to the hydro-meteorological event of 2015 . Similarly to the analysis performed using the populated places layer, the facilities datasets have been integrated in the system and overlaid to the hazard information.

Another layer that has been used is the Malawi educational buildings dataset. It contains the geographic location of schools and universities which may represent a fundamental asset in case of flooding event for essentially two reasons. Firstly, educational buildings for most of the day are populated by children and young students, which usually are the most affected population by flood. Secondly these structures can be used as gathering points for the displaced populations. Therefore an analysis of facilities potentially at risk allows decision makers to implement specific monitoring actions and to define promptly and timely targeted activities to safeguard the life of students. Moreover, an immediate identification of the structures suitable as collection points may eventually accelerate the rescue activities. Educational buildings affected, such as those located in the south/south-eastern part of the country, where several facilities resulted characterized by a high level of alert, are recognized (Fig. 7.4). Since emergency facilities, such as health-care structures, are particularly important during and after a disaster, the dataset containing their location has been downloaded from MASDAP and integrated in the system (Fig. 7.5).

The presence of these facilities might be an advantage but, however, their locations and the morphology of the surrounding environments sometimes may hamper their proper functioning.

A region characterized by several emergencies structures could be anyway very vulnerable if these facilities are located in an inappropriate area and appropriated operations of rescue teams and medical personnel might be not guaranteed in case of disaster. Therefore this analysis has been conducted integrating the Malawi Health dataset to the layer of the extreme precipitation alerts in order to understand which facilities resulted to be potentially affected after the flooding events of the 2015. Using these information, decision makers and local government personnel may define promptly and timely targeted actions to safeguard the human life. The identification of unaffected healthcare structures allows to better organize the emergency phases.

The last analysis on the potentially impacted features has been conducted considering the transportation system. Data retrieved from the Malawi road network dataset has been superimposed to the extreme precipitation alerts. The results of this analysis are sections of primary and secondary Malawi roads likely affected by extreme precipitations (Fig. 7.6). In this way, it is possible to understand which and where may be the possible road network interruptions. Thus, this cartographic product represents a strategic tool because it allows to identify probably isolated and unreachable settlements.

Moreover, the possibility to hold such information in near-real time and in the immediate aftermath of disastrous event allows to greatly improve the emergency 


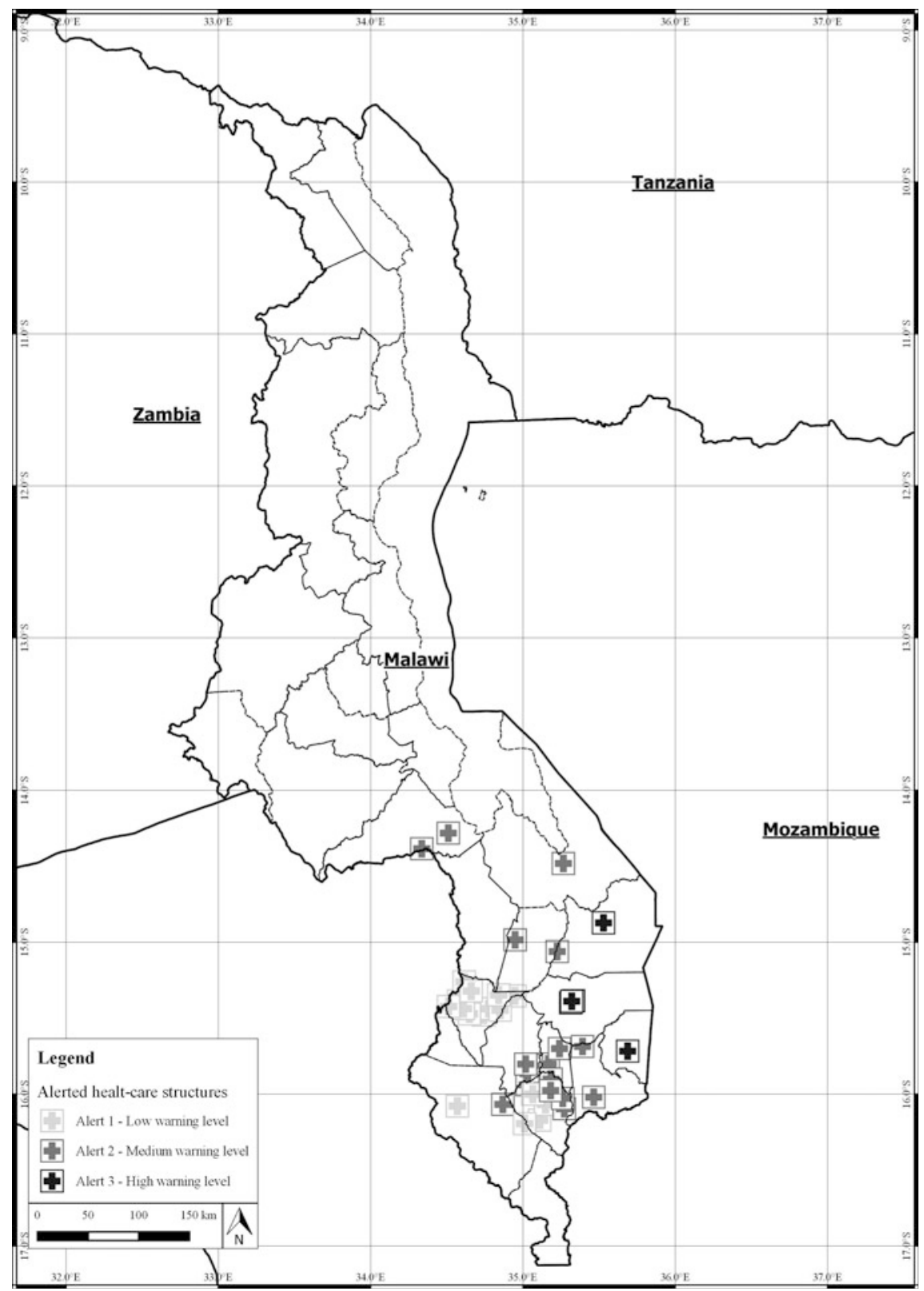

Fig. 7.4 Malawi educational buildings alerted on the basis of extreme precipitation alerts issued for the 12th January 2015 


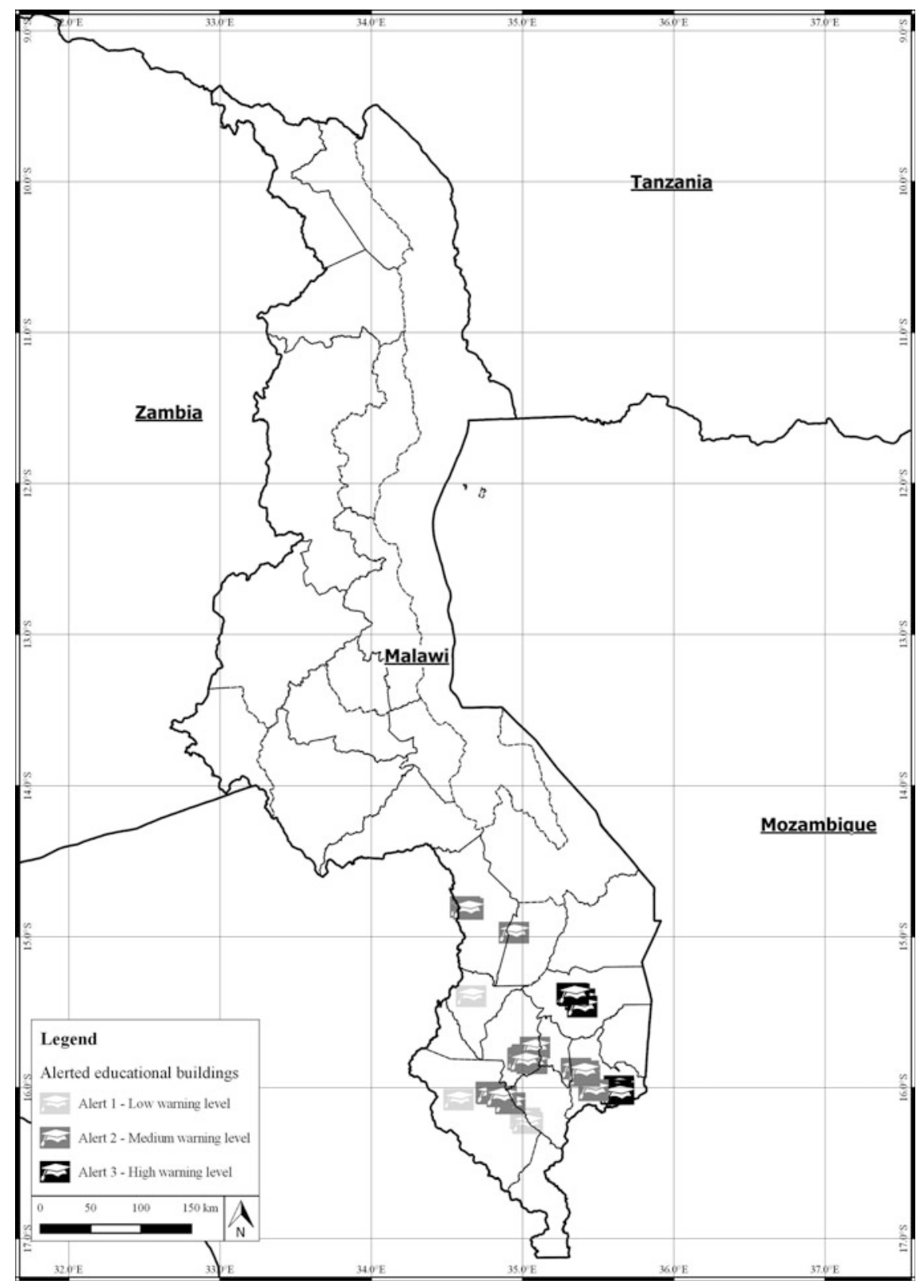

Fig. 7.5 Malawi health-care facilities alerted on the basis of extreme precipitation alerts issued for 12th January 2015 


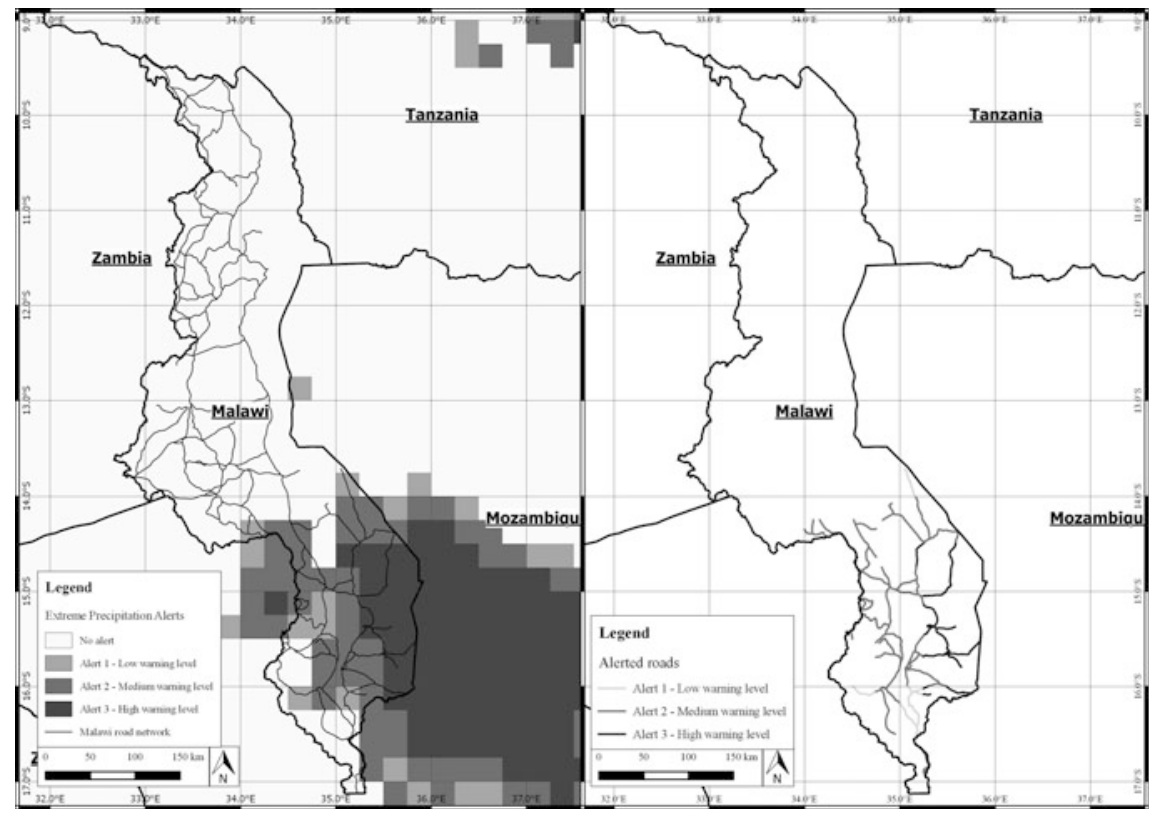

Fig. 7.6 Extreme precipitation alerts issued for the 12th January 2015 and the Malawi road network (on the left) and alerted section of the Malawi road network in relation to the amount of precipitation measured (on the right)

phases in terms of results and response time. Indeed, the possibility to plan in advance the routes to be used by rescue teams or to locate and pinpoint back-up network systems certainly allows to save great amount of resources, both in terms of time and money.

\subsection{Conclusions}

The methodology developed in this work is aimed at enhancing the adaptation and resilience to hydro-meteorological risks of local and urban communities. For this purpose, this paper presents a multidisciplinary approach to produce immediately understandable and easy to use information about the potential impacts generated by extreme rainfall events.

The methodology relies on the development of a tailored GIS that allows the integration, management and visualization of different types of information commonly used to perform the risk assessment (i.e. satellite derived precipitation estimates and geospatial datasets concerning the geographic features of the context analysed).

Simple hazard representations (i.e. near-real time accumulated precipitations) are very useful tools for a first delineation of the event in term of magnitude and spatial 
evolution. However, an accurate assessment of population, infrastructure and facilities at risk is necessary to enhance the response of local communities facing extreme hydro-meteorological risks. The possibility to easily identify the places and the assets potentially most at risk is thus a paramount functionality. Therefore, information concerning the geographic context (such as the populated places, the road network, the health-care facilities and the educational buildings) have been integrated along with extreme precipitation alerts to produce a final risk information immediately understandable by technical and non-technical people.

The methodology developed has been validated for the case study of the Malawi 2015 Flood event. Results clearly demonstrate that the methodology represents a valuable spatial decision support system tool for local decision makers during the emergency management and the DRR. Indeed, the created GIS has allowed firstly to geographically contextualize and to quantify the magnitude of extreme weather events. Secondly, it has enabled to translate results of weather predictions and scientific analysis into meaningful information for decision makers. Thirdly, it has allowed to identify and monitor possible impacts on communities. Fourthly, the developed tool has permitted to quickly produce easy-to-understand cartographic products able to address the evolution of hydro-meteorological events and the related potential impacts for the urban and local communities. Therefore, on the basis of the information provided by this methodology, decision makers and local government personnel may evaluate promptly and timely targeted actions, implementing all necessary measures to safeguard the life and property in areas at risk.

Moreover, the flexibility of the GIS environment enables the development of a repeatable and standardisable procedure that may be adapted to support a variety of actors involved in resilience and adaptation projects also in other geographic areas.

It should be noticed that this methodology relies on the use of open-source data in an open-source environment. Open-source geospatial information technologies allow to effectively reduce costs, both in term of time and economic resources, required for carrying out in situ spatial surveys. However, the accuracy of the alerts issued by this tool is strictly related on the resolution of the satellite imageries used. Using open-source data characterized by medium-low resolutions, this tool is suitable only for analyses at national and regional scale. Therefore, further developments of the research should consider the implementation of analyses at a higher resolution to cover local scale events. Moreover, including topographic information (i.e. DTM-Digital Terrain Model and DEM-Digital Elevation Model) and layers of historical floods, can significantly improve the reliability and accuracy of the methodology.

\section{References}

Altan, O., R. Backhaus, P. Boccardo, F. Giulio Tonolo, J. Trinder, N. Van Manen, and S. Zlatanova. 2013. The Value of Geoinformation for Disaster and Risk Management (VALID): Benefit Analysis and Stakeholder Assessment. Joint Board of Geospatial Information Societies (JBGIS): Copenhagen. 
Balbo, S., P. Boccardo, S. Dalmasso, and P. Pasquali. 2013. A public platform for geospatial data sharing for disaster risk management. In International Society for Photogrammetry and Remote Sensing (ISPRS) Archives, ed. F. Pirotti, A. Guarnieri, and A. Vettore: 189-195, Padua, Italy. XL-5/W3, W (The role of geomatics in hydrogeological risk, 27-28 February 2013.

Bonetto, S., A. Facello, E.I. Cristofori, W. Camaro, and A. Demarchi. 2016. An approach to use earth observation data as support to water management issues in the Ethiopian rift. In: Symposium on climate change adaptation in Africa 2016-Addis Ababa Ethiopia. 2016.

Cardona, O.D., M.K. van Aalst, J. Birkmann, M. Fordham, G. McGregor, R. Perez, R.S. Pulwarty, E.L.F. Schipper, and B.T. Sinh. 2012. Determinants of risk: exposure and vulnerability. In Managing the risks of extreme events and disasters to advance climate change adaptation. A special report of working groups I and II of the Intergovernmental Panel on Climate Change (IPCC), ed. C.B. Field, V. Barros, T.F. Stocker, D. Qin, D.J. Dokken, K.L. Ebi, M. D. Mastrandrea, K.J. Mach, G.K. Plattner, S.K. Allen, M. Tignor, and P.M. Midgley, 65-108. Cambridge, UK, and New York, USA: Cambridge University Press.

Cay, T., F. Iscan, and S.S. Durduran. 2004. The cost analysis of satellite images for using in GIS by the pert. In XX ISPRS Congress, Com. IV, 12-23 July 2004, XXXV(B4). 2004, Istanbul, pp. 358-363.

CRED - Centre for Research on the Epidemiology of Disasters and UNISDR-United Nations Office for Disaster Risk Reduction. 2016. The Human Cost of Weather-related Disasters 1995 2015. http://www.emdat.be/publications.

Chigbu, N. and D. Onukaogu. 2013. Role of geospatial technology in environmental sustainability in Nigeria-An overview. In FIG working week 2013 environment for sustainability. Abuja, Nigeria, 6-10 May, 2013.

Cristofori, E.I., A. Albanese, and P. Boccardo. 2016a. Early warning systems \& geomatics: Value-added information in the absence of high resolution data. In Planning to cope with tropical and subtropical climate change. ed. M. Tiepolo, E. Ponte, E. Cristofori, 141-153. doi:0.1515/9783110480795-009.

Cristofori, E.I., S. Balbo, W. Camaro, P. Pasquali, P. Boccardo, and A. Demarchi. 2015. Flood risk web-mapping for decision makers: A service proposal based on satellite-derived precipitation analysis and geonode. In IEEE International Geoscience and Remote Sensing Symposium, IGARSS 2015 (2015): 1389-1392.

Cristofori, E.I., A. Facello, A. Demarchi, W. Camaro, M. Fascendini, and A. Villanucci. 2016b. A geographic information system as support to the healthcare services of nomadic community, the Filtu Woreda case study. In: Symposium on climate change adaptation in Africa 2016Addis Ababa Ethiopia. 2016.

Donoghue, D.N.M. 2002. Remote sensing: Environmental change. Physical Geography 26 (1): $144-151$.

Duncan, M., K. Crowley, R. Cornforth, S. Edwards, R. Ewbank, P. Karbassi, C. McLaren, J.L. Penya, A. Obrecht, S. Sargeant, and E. Visman. 2014. Integrating science into humanitarian and development planning and practice to enhance community resilience. Full guidelines.

Ehrlich, D., and P. Tenerelli. 2012. Optical satellite imagery for quantifying spatio-temporal dimension of physical exposure in disaster risk assessments. Natural Hazards 68 (3): 1271-1289. doi:10.1007/s11069-012-0372-5.

Eiser, J.R. and White et al. 2012. Risk interpretation and action: a conceptual framework for responses to natural hazards. International Journal of Disaster Risk Reduction 1: 5-16. doi:10.1016/j.ijdrr.2012.05.002.

Ferrandino, J. 2014. Incorporating GIS as an interdisciplinary pedagogical tool throughout an MPA program. Journal of Public Affairs Education 20 (4): 529-544.

Government of Malawi. 2015. Malawi 2015 floods post disaster needs assessment report Malawi 2015 floods.

Huffman, G.J., R.F. Adler, D.T. Bolvin, G. Gu, E.J. Nelkin, K.P. Bowman, Y. Hong, E.F. Stocker, and D.B. Wolff. 2007. The TRMM multisatellite precipitation analysis (TMPA): Quasi-global, multiyear, combined-sensor precipitation estimates at fine scales. Journal of Hydrometeorology 8 (1): $38-55$. 
Huffman, G.J., R.F. Adler, D.T. Bolvin, and E.J. Nelkin. 2010. The TRMM multi-satellite precipitation analysis (TMPA). In Satellite rainfall applications for surface hydrology, ed. F. Hossain, and M. Gebremichael, 3-22. Springer.

IPCC-Intergovernmental Panel on Climate Change. 2014. Contribution of Working Group II to the Fifth Assessment Report on Climate Change 2013: The Physical Science Basis. Cambridge: United Kingdom.

IPCC. 2012. Managing the risks of extreme events and disasters to advance climate change adaptation. A special report of working groups I and II of the Intergovernmental Panel on Climate Change. Cambridge, United Kingdom.

Melesse, A.M., Q. Weng, P.S. Thenkabail, and G.B. Senay. 2007. Remote sensing sensors and applications in environmental resources mapping and modelling. Sensors 7 (12): 3209-3241. doi:10.3390/s7123209.

Miyazaki, H., M. Nagai, and R. Shibasaki. 2015. Reviews of geospatial information technology and collaborative data delivery for disaster risk management. ISPRS International Journal of Geo-Information 4 (4): 1936-1964.

Pandey, J., and D. Pathak. 2014. Geographic Information System. New Delhi: The Energy and Resources Institute, TERI.

Sundaresan, J., K.M. Santosh, A. Déri, R. Roggema, and R. Singh. 2014. Geospatial Technologies and Climate Change. Springer International Publishing.

Teegavarapu, R.S.V. 2012. Floods in a changing climate. Extreme precipitation. New York: Cambridge University Press.

Tiepolo, M., and E.I. Cristofori. 2016a. Climate change characterisation and planning in large tropical and subtropical cities. In Planning to cope with tropical and subtropical climate change, ed. M. Tiepolo, E. Ponte, and E. Cristofori, 6-41. Berlin: De Gruyter Open.

Tiepolo, M., and E. Cristofori. 2016b. Planning the adaptation to climate change in cities: an introduction. In Planning to Cope with Tropical and Subtropical Climate Change, ed. M. Tiepolo, E. Ponte, and E. Cristofori, 1-5. Berlin: De Gruyter Open.

Tomaszewski, B. 2014. Geographic Information Systems (GIS) for Disaster Management. Boca Raton: CRC Press.

UNISDR. 2015. Making development sustainable: the future of disaster risk management. Global assessment report on disaster risk reduction. Geneva, Switzerland: United Nations Office for Disaster Risk Reduction.

UNDESA-United Nations Department of Economic and Social Affairs, Population Division. 2011. World Population Prospects: The 2010 Revision, Highlights and Advance Tables.

Open Access This chapter is licensed under the terms of the Creative Commons Attribution 4.0 International License (http://creativecommons.org/licenses/by/4.0/), which permits use, sharing, adaptation, distribution and reproduction in any medium or format, as long as you give appropriate credit to the original author(s) and the source, provide a link to the Creative Commons license and indicate if changes were made.

The images or other third party material in this chapter are included in the chapter's Creative Commons license, unless indicated otherwise in a credit line to the material. If material is not included in the chapter's Creative Commons license and your intended use is not permitted by statutory regulation or exceeds the permitted use, you will need to obtain permission directly from the copyright holder.

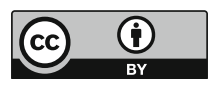

